\title{
An Analytical Calculation of the Axial Density Profile for 1-D Slab Expansion
}

\author{
D. Ho
}

\section{August 10, 1999}

U.S. Department of Energy

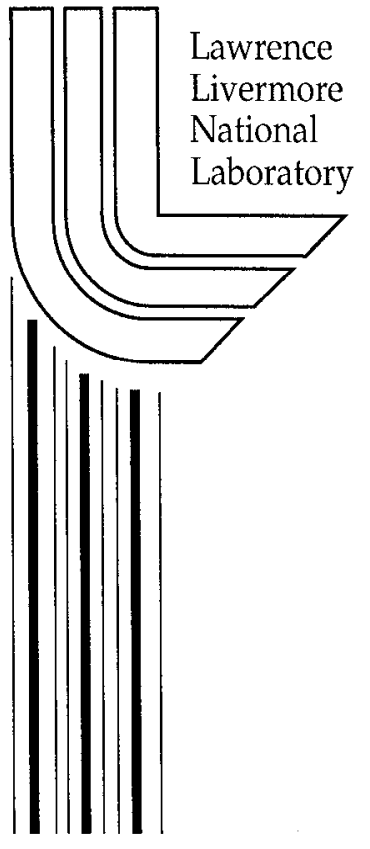




\section{DISCLAIMER}

This document was prepared as an account of work sponsored by an agency of the United States Government. Neither the United States Government nor the University of California nor any of their employees, makes any warranty, express or implied, or assumes any legal liability or responsibility for the accuracy, completeness, or usefulness of any information, apparatus, product, or process disclosed, or represents that its use would not infringe privately owned rights. Reference herein to any specific commercial product, process, or service by trade name, trademark, manufacturer, or otherwise, does not necessarily constitute or imply its endorsement, recommendation, or favoring by the United States Government or the University of California. The views and opinions of authors expressed herein do not necessarily state or reflect those of the United States Government or the University of California, and shall not be used for advertising or product endorsement purposes.

Work performed under the auspices of the U. S. Department of Energy by the University of California Lawrence Livermore National Laboratory under Contract W-7405-Eng-48.

This report has been reproduced directly from the best available copy.

Available to DOE and DOE contractors from the Office of Scientific and Technical Information

P.O. Box 62, Oak Ridge, TN 37831

Prices available from (423) 576-8401

http://apollo.osti.gov/bridge/

Available to the public from the National Technical Information Service

U.S. Department of Commerce 5285 Port Royal Rd., Springfield, VA 22161 http://www.ntis.gov/

OR

Lawrence Livermore National Laboratory Technical Information Department's Digital Library http://www.llnl.gov/tid/Library.html 


\title{
An Analytical Calculation of the Axial Density Profile for 1-D Slab Expansion
}

\author{
by \\ Darwin D.-M. IIo \\ Lawrence Livermore National Laboratory
}

\section{Summary}

Obtaining an analytical expression for the axial density profile can provide us with a quick and convenient way to evaluate the density evolution for targets with different densities and dimensions. In this note, we show that such an analytical expression can be obtained based on the self-similar solutions and the method of characteristics for 1-D slab expansion.

\section{Introduction}

For a slab target inside a tube, the expansion is 1-D along the axis if the electron energy deposition on the target is uniform in the radial direction and if the target is not very thick. Then, to the lowest order of approximation, the energy deposition can be assumed to be uniform in the axial direction. Under these conditions, an analytical expression for the temporal evolution of the axial density profile can be obtained. The schematics of the density profile evolution for such slab target expansion is shown in Fig. 1.

'The initial configuration of the slab has a thickness of $2 \mathrm{~L}$ and has density $\rho_{0}$ as shown in Fig. 1a. Here, we assume that temperature of the slab is raised to its final value $\mathrm{T}_{\mathrm{f}}$ instantaneously. If the slab is composed of an ideal gas with $\gamma=5 / 3$, then the front and back surfaces of the slab have an expansion velocity of $3 \mathrm{c}_{0}$ [Ref. 1], where $\mathrm{c}_{0}$ is the sonic velocity corresponding to the slab at $\rho_{0}$ and $T_{f}$, see Fig. $1 b$. These surfaces expanding at $3 \mathrm{c}_{0}$ are referred to as the "tails" of the rarefaction wave. Meanwhile, the "heads" of the rarefaction waves are moving into the interior region of the slab at $\mathrm{c}_{0}$. At time $t=\mathrm{L} / \mathrm{c}_{0}$, the heads of the rarefaction wave meet at the center of the slab as shown in Fig. 1c. For $t>$ $\mathrm{L} / \mathrm{c}_{0}$, the heads of the rarefaction waves have passed each other and the overlapping region is called the "nonsimple wave region" as shown in Fig. 1d. The regions where the waves do not overlap each other are called "simple wave region".

In the simple wave region, the analytical solution for the evolution of the fluid variables are well known and are available in many text books of fluid mechanics [Refs. 1 - 3]. However, there are no analytical solutions for the fluid variables in the nonsimple wave region. The primary objective for this note is to derive an approximate, but accurate, analytical solution in the nonsimple wave region. 


\section{Derivation of the approximation solution for the nonsimple wave region}

In order to derive the approximation solution for the nonsimple wave region, we need to look at the behavior of the characteristics of an expanding slab as shown in Fig. 2. Only the left-hand-half of the slab is shown here. For $t>t_{0} \equiv L / c_{0}$ or $c_{0} t / L>1$, the characteristics launched toward the interior of the slab from the surfaces on the left-and right-hand side overlap and form the nonsimple wave region. (Note that Fig.2 is for the expansion of a slab of ideal gas with $\gamma=2$. Based on this figure, the approximation that we use for the nonsimple wave region, as discussed later in this section, applies to ideal gas with $\gamma=5 / 3$ as well.)

The self-similar solutions that describe the density $\rho$ and axial velocity $v$ in the simple wave region are:

$$
\begin{aligned}
& \frac{\rho}{\rho_{0}}=\left\{\begin{array}{lll}
1 & \mathrm{z}>\mathrm{c}_{0} \mathrm{t}-\mathrm{L}, & 0<\mathrm{t}<\mathrm{t}_{0} \\
\left(\frac{3}{4}+\frac{1}{4} \frac{\mathrm{z}+\mathrm{L}}{\mathrm{c}_{0} \mathrm{t}}\right)^{3} & \begin{cases}-3 \mathrm{c}_{0}-\mathrm{L}<\mathrm{z}<\mathrm{c}_{0} \mathrm{t}-\mathrm{L}, & 0<\mathrm{t} \leq \mathrm{t}_{0} \\
-3 \mathrm{c}_{0}-\mathrm{L}<\mathrm{z}<\mathrm{z}_{0}, & \mathrm{t}>\mathrm{t}_{0}\end{cases} \\
0 & \mathrm{z}>-3 \mathrm{c}_{0}-\mathrm{L}, & \mathrm{t}>0
\end{array}\right. \\
& \frac{\mathrm{v}}{\mathrm{c}_{0}}= \begin{cases}0 & \mathrm{z}>\mathrm{c}_{0} \mathrm{t}-\mathrm{L}, 0<\mathrm{t} \leq \mathrm{t}_{0} \\
-\frac{3}{4}\left(1-\frac{\mathrm{z}+\mathrm{L}}{\mathrm{c}_{0} \mathrm{t}}\right) & \begin{cases}-3 \mathrm{c}_{0} \mathrm{t}-\mathrm{L}<\mathrm{z}<\mathrm{c}_{0} \mathrm{t}-\mathrm{L}, & 0<\mathrm{t}<\mathrm{t}_{0} \\
-3 \mathrm{c}_{0} \mathrm{t}-\mathrm{L}<\mathrm{z}<\mathrm{z}_{\mathrm{b}}, & \mathrm{t}>\mathrm{t}_{0}\end{cases} \end{cases}
\end{aligned}
$$

Curve ab in Fig. 2 is the boundary that separates the simple and nonsimple wave regions. The expression that describes this boundary as a function of time $t$ has the form [Ref. 3, p. 391. But be careful, the equation given in Ref. 3 has an error!]

$$
z_{b}=-3 c_{0} t+4 L\left(\frac{t}{t_{0}}\right)^{\frac{1}{2}}-L
$$

Combining Eqs. (1) and (3) gives the density $\rho_{b}$ along the boundary.

$$
\rho_{\mathrm{b}}=\rho_{0} \xi^{-\frac{3}{2}}
$$

where $\xi \equiv \mathrm{c}_{0} \mathrm{t} / \mathrm{L}=\mathrm{t} / \mathrm{t}_{0}$. boundary,

Combing Eqs. (2) and (3) gives the expression for the fluid velocity $v_{b}$ along the 


$$
\frac{v_{b}}{c_{0}}=-3\left(1-\xi^{-1 / 2}\right)
$$

Inspection of the characteristics in the nonsimple region shows that the characteristics can be approximated by straight lines as long as $\mathrm{c}_{0} \mathrm{t} / \mathrm{L}$ is not substantially greater than unity. This implies that $v$ vs $z$ is also a straight line in the nonsimple wave region (see Fig. 3), e.g.

$$
\frac{v(z, t)}{c_{0}}=\frac{v_{b}\left(z_{b}, t\right) z}{c_{0} z_{b}}
$$

Substitute Eqs. (3) and (5) into the above expression, we obtain

$$
\frac{v(z, t)}{c_{0}}=\frac{-3\left(1-\xi^{-1 / 2}\right) z}{L\left(-1-3 \xi+4 \xi^{1 / 2}\right)}
$$

To obtain the density as a function of $z$ and $t$, we need to substitute Eq. (7) into momentum equation

$$
\frac{\partial \mathrm{v}}{\partial \mathrm{t}}+\mathrm{v} \frac{\partial \mathrm{v}}{\partial \mathrm{z}}=-\frac{1}{\rho} \frac{\partial \mathrm{P}}{\partial \mathrm{z}}
$$

To relate $\rho$ and the pressure $\mathrm{P}$, we combine the expression for the sonic velocity $\mathrm{c}^{2}=\gamma \mathrm{P} / \rho$ and the adiabatic relation $\mathrm{P}=$ const. $\rho^{\gamma}$ and obtain

$$
\mathrm{P}=\frac{\mathrm{c}_{0}{ }^{2}}{\gamma \rho_{0}{ }^{\gamma-1}} \rho^{\gamma}
$$

Taking the derivative, Eq. (9) becomes

$$
-\frac{1}{\rho} \frac{\partial \mathrm{P}}{\partial \mathrm{z}}=-\frac{\mathrm{c}_{0}{ }^{2}}{(\gamma-1) \rho_{0}^{\gamma-1}} \frac{\partial \rho^{\gamma-1}}{\partial \mathrm{z}}
$$

Substitute Eqs. (7) and (10) into Eq. (8) and integrating from an arbitrary location $\mathrm{z}$ inside the nonsimple wave region to the boundary $z_{b}$, the individual terms in the momentum equation become

$$
\int_{z}^{z_{b}} \frac{\partial \mathrm{v}}{\partial \mathrm{t}} \mathrm{d} z=\frac{1}{2}\left(\frac{\mathrm{c}_{0}}{\mathrm{~L}}\right)^{2}\left(\frac{3}{2} \xi^{-3 / 2}-12 \xi^{-1}+\frac{39}{2} \xi^{-1 / 2}-9\right) \frac{\mathrm{z}_{\mathrm{b}}{ }^{2}-\mathrm{z}^{2}}{\left(-1-3 \xi+4 \xi^{1 / 2}\right)^{2}}
$$




$$
\begin{aligned}
& \int_{z}^{i_{b}} \mathrm{v} \frac{\partial \mathrm{v}}{\partial \mathrm{z}} \mathrm{dz}=\frac{9}{2}\left(\frac{\mathrm{c}_{0}}{\mathrm{~L}}\right)^{2} \frac{\left(1-\xi^{-1 / 2}\right)^{2}\left(\mathrm{z}_{\mathrm{b}}{ }^{2}-\mathrm{z}^{2}\right)}{\left(13 \xi+4 \xi^{1 / 2}\right)^{2}} \\
& \int_{z}^{z_{b}}-\frac{1}{\rho} \frac{\partial \rho}{\partial z} \mathrm{~d} z=-\frac{3}{2} \frac{\mathrm{c}_{0}{ }^{2}}{\rho_{0}{ }^{2 / 3}}\left(\rho_{\mathrm{b}}{ }^{2 / 3}-\rho^{2 / 3}\right)
\end{aligned}
$$

Adding Eqs. (11) and (12) and then equate the sum to Eq. (13), the expression for the density $\rho$ as a function of $z$ and $t$ in the nonsimple wave region can then be oblained and has the form

$$
\frac{\rho}{\rho_{0}}=\left\{\xi^{-1}+\left(\frac{\xi^{-3 / 2}}{2}-\xi^{-1}+\frac{\xi^{-1 / 2}}{2}\right)\left[1-\frac{\left(\mathrm{z}_{\mathrm{b}}{ }^{2}-\mathrm{z}^{2}\right) / \mathrm{L}^{2}}{\left(-1-3 \xi+4 \xi^{1 / 2}\right)^{2}}\right]\right\}^{3 / 2}
$$

The accuracy of this expression can be checked by comparing the total charge inside the nonsimple-wave region calculated by Eq. (16) to the result obtained by subtracting the charge in the simple-wave region obtained from Eq. (1) from the total charge. This shows excellent agreement; e.g., at $t=2 t_{0}$, the agreement is within $1 \%$. Another way to verify the accuracy of Eq. (16) is by substituting this expression into the continunity equation. We find that the cancellation between the $\partial \rho \partial t$ term and the $(\partial \partial \mathrm{z})(\mathrm{v} \rho)$ terms in the continunity equation is within $3 \%$ of the $\partial \rho \partial \mathrm{t}$ term at $\mathrm{z}=0$ and $\mathrm{t}=$ $2 t_{0}$. The shape of the density profile described by Eq. (16) is also in close agreement with that obtained from PIC simulations (Ref. 4). However, the errors resulting from the approximate solutions increase with time. For example, at $t=3 \mathrm{t}_{0}$, the value of the charge inside the nonsimple-wave region obtained from the approximate formula differs from the actual value by about $2 \%$. This suggest that Eq. (16) is able to approximate the beam density profile with good accuracy at least up to $t=3 t_{0}$.

\section{Numerical examples}

We study the expansion of slab targets inside a tube with different initial densitics but with the same mass and the same input energy. The energy is assumed to be deposited instantaneously. Here, the sonic velocity is independent of the initial density of the slabs since the final temperature $T_{\mathrm{f}}$ is independent of the density. The reason that we have a slab target inside a tube is because if the beam intensity is radially uniform and if the energy deposition is independent of the depth, the expansion is one dimensional in the axial direction and Eq. (16) can be applied.

The first example is a solid target with initial density $\rho_{0}$ and thickness $2 \mathrm{~L}$. The corresponding sonic velocity is $\mathrm{c}_{0}$. At $\mathrm{t}=2 \mathrm{t}_{0}$, the tail of the rarefaction wave has already covered a distance of $6 \mathrm{~L}$ since the tail of the wave is moving at $3 \mathrm{c}_{0}$. In the central region of the slab, the heads of the waves have already passed each other and created the 
nonsimple wave region. The density profiles in the simple wave region and the nonsimple wave region are calculated using Eqs. (1) and (16), respectively. The density profile at this time is shown in Fig. 4 a.

The second example is a foamed target with initial density $\rho_{0} / 3$ and thickness $6 \mathrm{I}$, since the mass of this target is the same as that of the solid target. Here, we assume that the total energy deposition is the same as that of the solid target and the energy is distributed uniformly over the entire thickness of 6L. Consequently, the temperature and the sonic velocity for this foamed target (before it expands) are the same as those for the solid target. At $t=1.33 \mathrm{t}_{0}$ (and at $\mathrm{t}=2 \mathrm{t}_{0}$ ), the tail of the rarefaction wave has covered a distance of $4 \mathrm{~L}(6 \mathrm{~L})$ and the head of the rarefaction has traveled a distance of $1.33 \mathrm{~L}$ ( $2 \mathrm{~L}$ ) to the right from the original edge of the slab at $-3 \mathrm{I}$. Since the heads of the rarefaction waves launched from the left and right edges of the slab have not met at the center at this time, the entire density profile consists of simple wave region and the undisturbed region at the center. The density profiles at $t=1.33 \mathrm{t}_{0}$ and $t=2 \mathrm{t}_{0}$ are shown in Figs. $4 \mathrm{~b}$ and $4 \mathrm{c}$, respectively.

The examples illustrated in Fig. 4 shown us that in comparing the plasma expansion of the solid and foamed targets, it is important to specify the criterion for comparison. For example, if we compare the distance traveled by the tail of the rarefaction wave from the center of the slab for the two cases, then the tail of the wave at anytime is further away from the center of the slab for the foamed target than the solid target. However, if we want to compare the plasma densities at a specified distance from the edge of the targets, then the solid target gives a higher plasma density than the foamed target at any given time. This is simply because the solid target has a higher initial density than the foamed target.

\section{Conclusion}

We have shown that the analytical expression for the axial density profile derived in this note is accurate for 1-D slab expansion. Using this expression, one can calculate the density evolution quickly. Results obtained from this expression improve our understanding of the expansion process.

Work performed under the auspices of the U.S. Department of Energy by Lawrence Livermore National Laboratory under contract No. W-7405-Eng-48.

\section{References}

(1) Y. B. Zel'Dovich and Y. P. Raizer, Physics of shock Waves and High-Temperature Hydrodynamic Phenomena (Academic Press, New York, 1966).

(2) L. D. Landau and E. M. Lifshitz, Fluid Mechanics (Pergamon Press, Oxford, 1959).

(3) A. H. Shapiro, The Dynamics and Thermodynamics of Compressible Fluid Flow (Ronald, New York, 1953). 
(4) D. D.-M. Ho, S. T. Brandon, and E. P. Lee, Particle Accelerators, 35, 15 (1991).

\section{Figure Captions}

Fig. 1 Evolution of density profile for a 1-D slab cxpansion

Fig. 2 Characteristics of ideal gas with $\gamma=2$

Fig. 3, Density and velocity vs $z$

Fig. 4 Density profiles

(a) solid target, $t=2 t_{0}$

(b) foamed target with $1 / 3$ solid target density, $t=1.33 \mathrm{t}_{0}$

(c) foamed target with $1 / 3$ solid target density, $t=2 t_{0}$ 
(a) $\mathrm{t}=0$

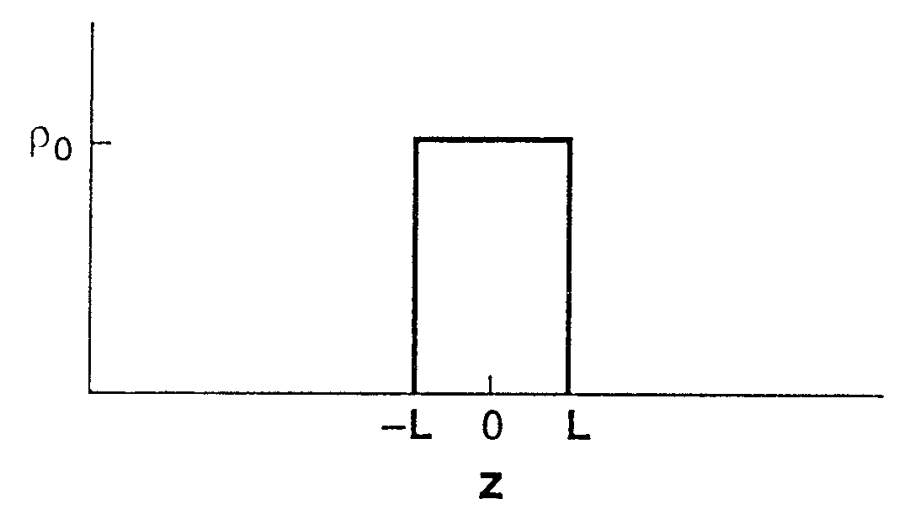

(c) $t=L / c_{0}$

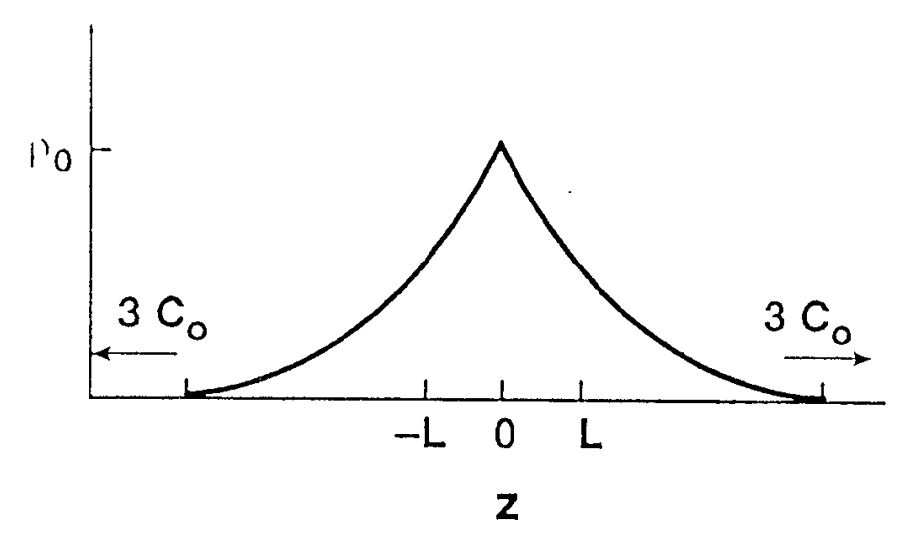

(b)

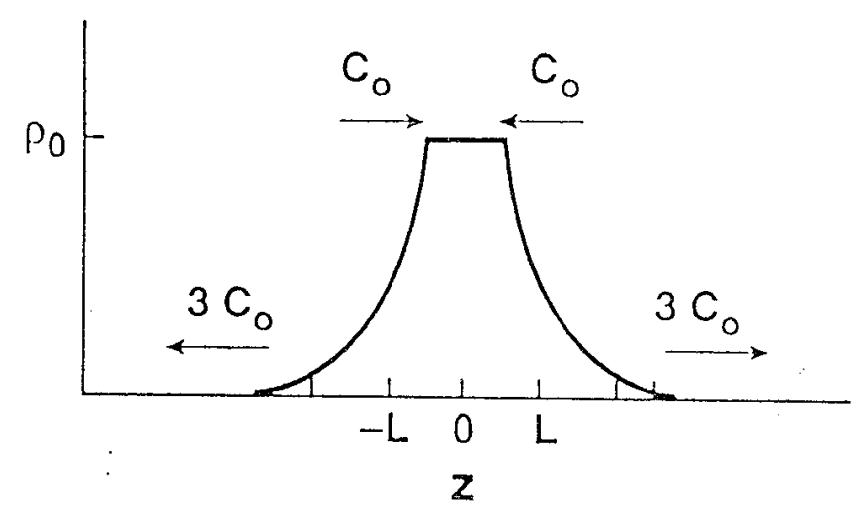

(d)

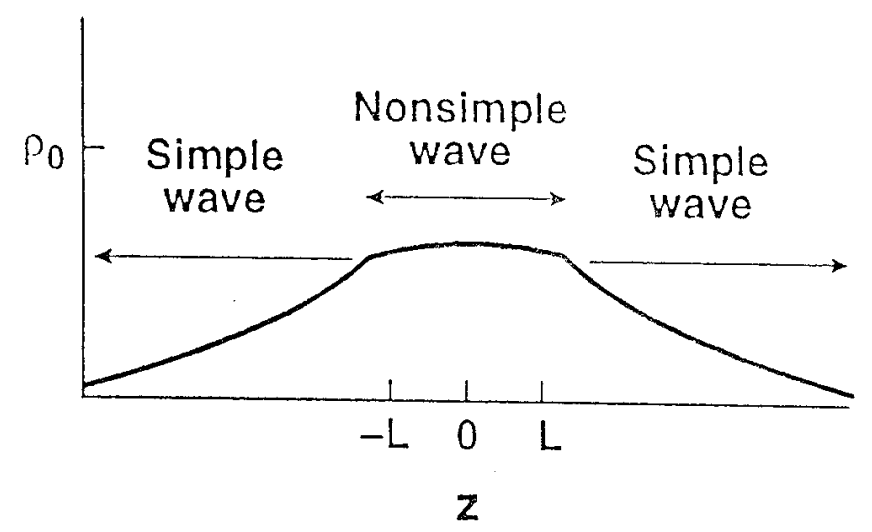

Fig. 1 


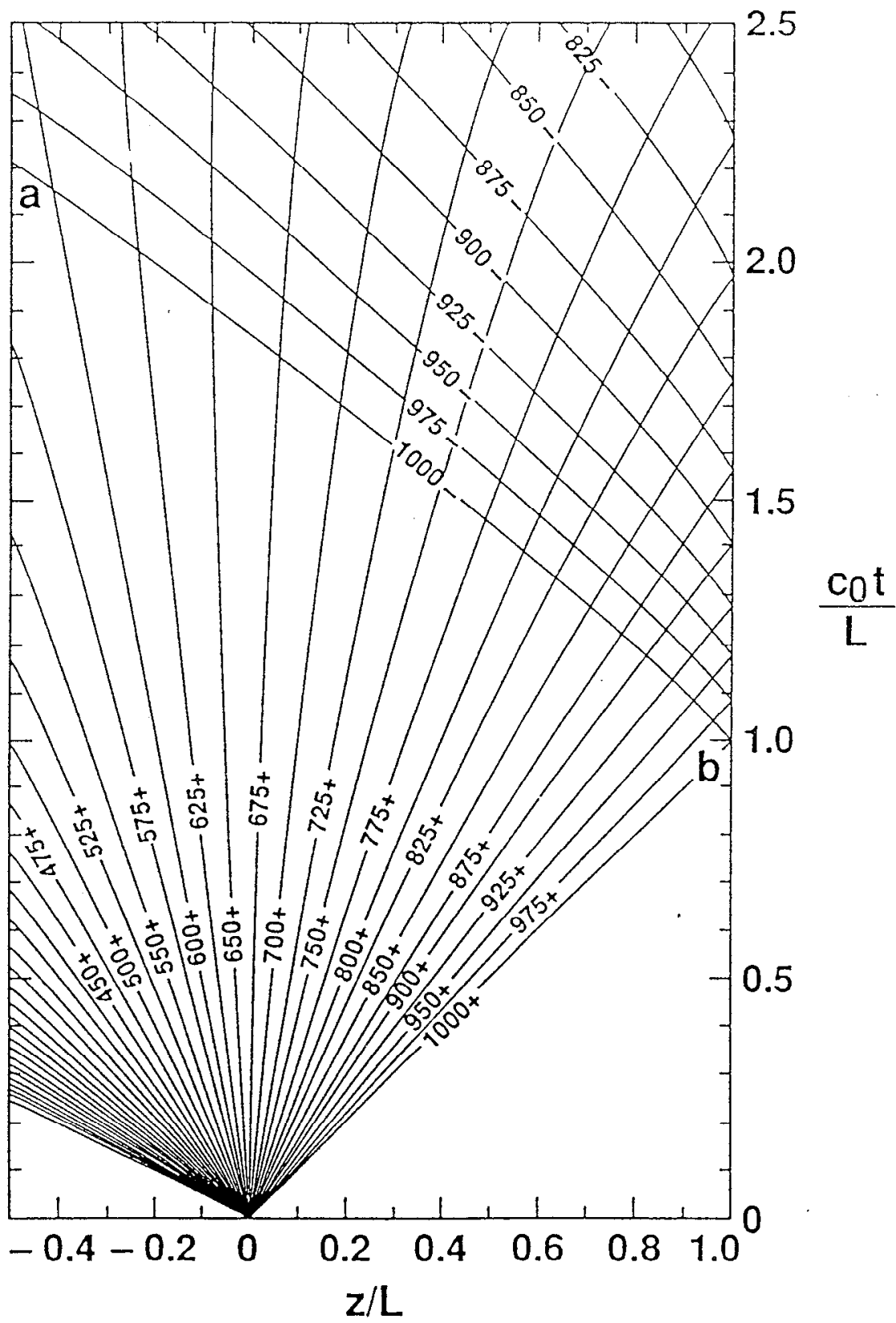

Fig. 2 

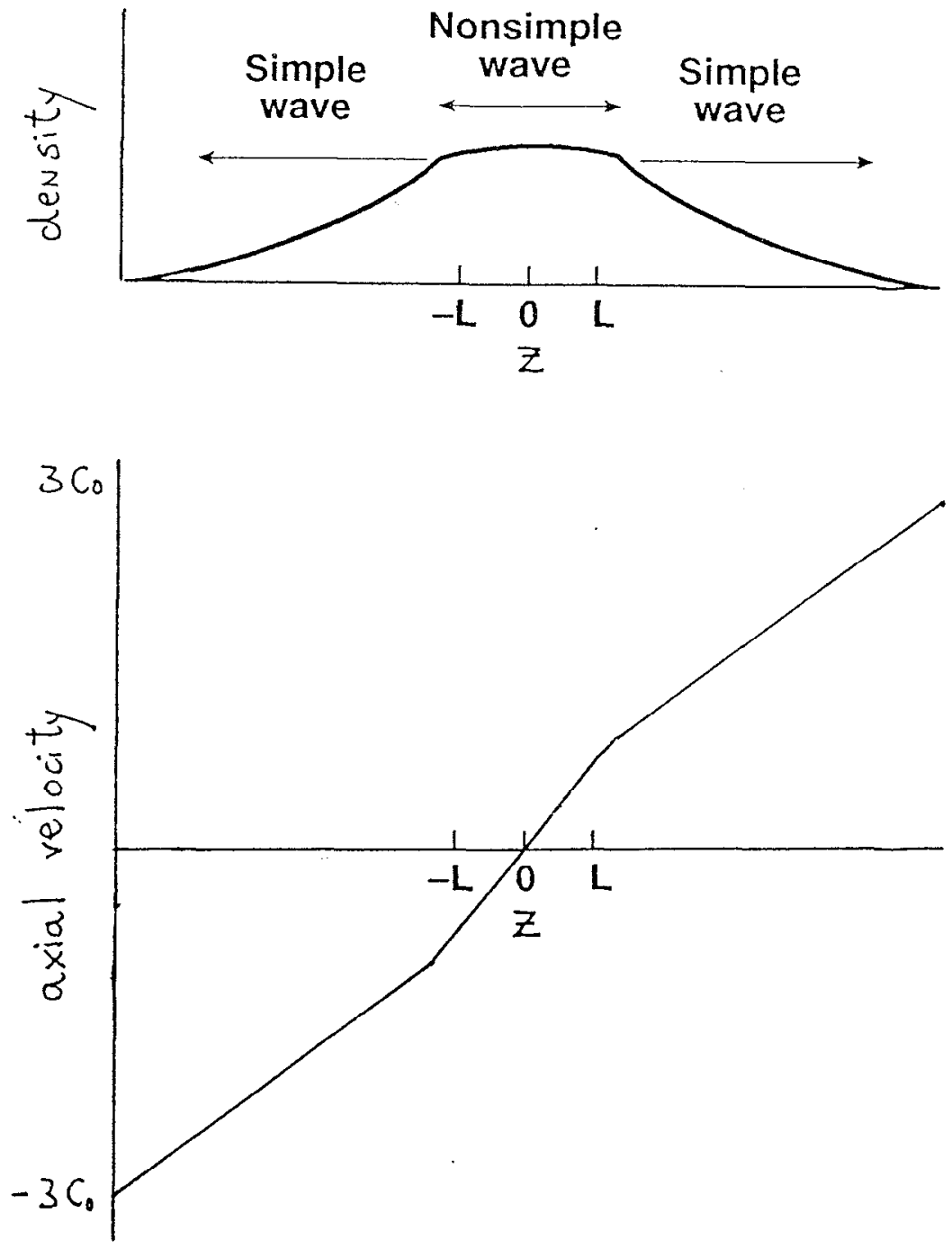

Fig. 3 
(a) Solid target $t=2 t_{0}$

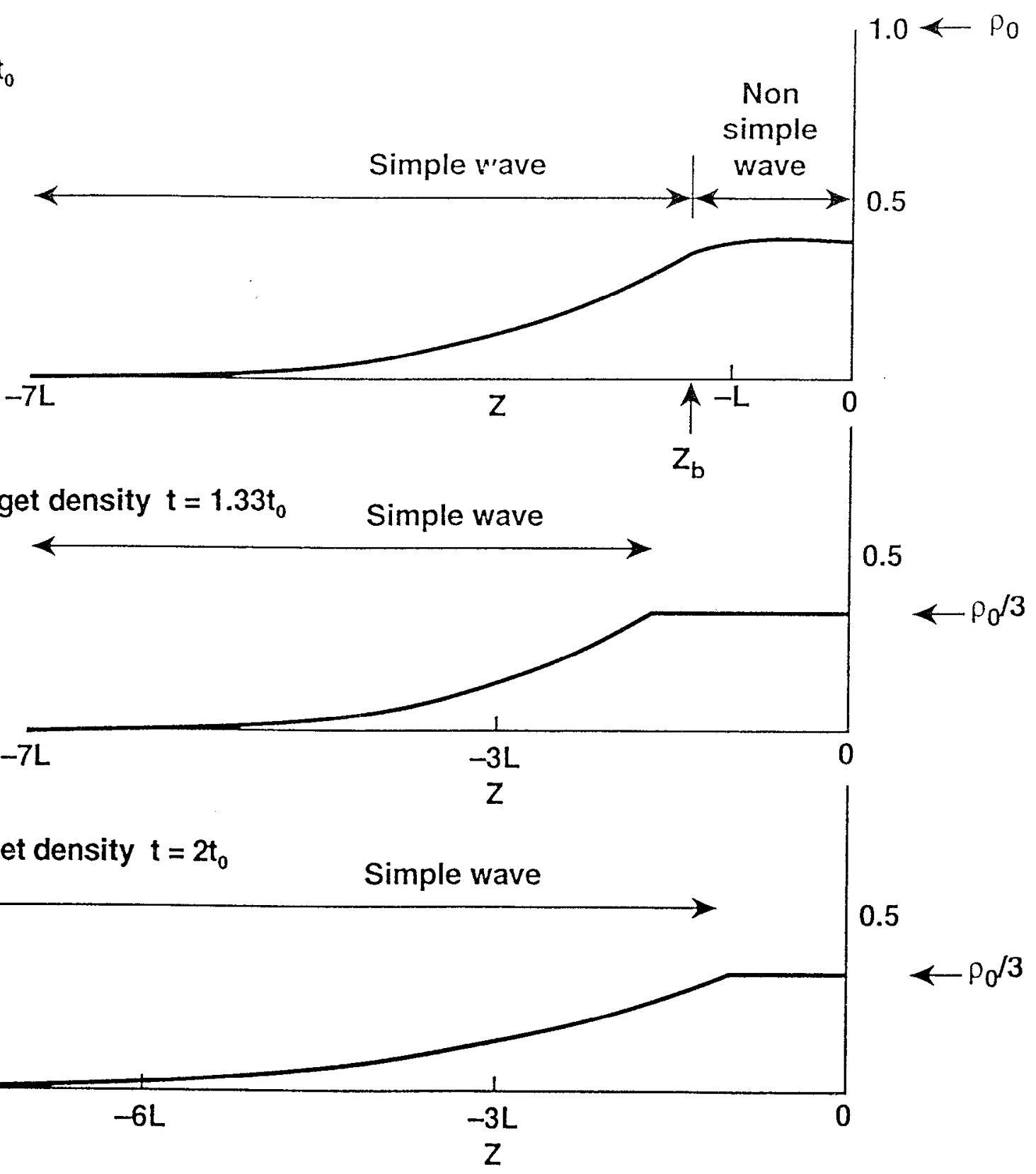

Fig. 4 